\title{
PROBING THE INTERGALACTIC MEDIUM WITH FAST RADIO BURSTS
}

\author{
Z. Zheng ${ }^{1}$, E. O. OfeK ${ }^{2}$, S. R. KULKARNi ${ }^{3}$, J. D. NeILL ${ }^{4}$, AND M. JURiC ${ }^{5}$ \\ ${ }^{1}$ Department of Physics \& Astronomy, University of Utah, 115 South 1400 East \#201, Salt Lake City, UT 84112, USA \\ ${ }^{2}$ Department of Particle Physics \& Astrophysics, Weizmann Institute of Science, Rehovot 76100, Israel \\ ${ }^{3}$ Caltech Optical Observatories 249-17, California Institute of Technology, Pasadena, CA 91125, USA \\ ${ }^{4}$ Space Radiation Laboratory 290-17, California Institute of Technology, Pasadena, CA 91125, USA \\ 5 Department of Astronomy, University of Washington, Box 351580, Seattle, WA 98195, USA \\ Received 2014 September 9; accepted 2014 October 13; published 2014 November 25
}

\begin{abstract}
The recently discovered fast radio bursts (FRBs), presumably of extragalactic origin, have the potential to become a powerful probe of the intergalactic medium (IGM). We point out a few such potential applications. We provide expressions for the dispersion measure and rotation measure as a function of redshift, and we discuss the sensitivity of these measures to the He II reionization and the IGM magnetic field. Finally, we calculate the microlensing effect from an isolated, extragalactic stellar-mass compact object on the FRB spectrum. The time delays between the two lensing images will induce constructive and destructive interference, leaving a specific imprint on the spectra of FRBs. With a high all-sky rate, a large statistical sample of FRBs is expected to make these applications feasible.
\end{abstract}

Key words: cosmology: miscellaneous - intergalactic medium - pulsars: general - radio continuum: general

\section{INTRODUCTION}

Variability of cosmological radio sources has long been proposed to probe the properties of the intergalactic medium (IGM). Haddock \& Sciama (1965) suggested to detect IGM dispersion measure (DM) through the variability of the radio signal from quasars, aiming at using the inferred DM to distinguish different cosmological models. Weinberg (1972) and Ginzburg (1973) suggested the use of radio flares to measure the DM and thus probe the IGM density. Later, radio emission from gamma-ray bursts (GRBs) and their afterglows were proposed as means to determine distances to GRBs and to probe the IGM (Palmer 1993), to study the prehistory of GRBs (Lipunova et al. 1997), and to constrain the hydrogen reionization history of the universe (Ioka 2003; Inoue 2004). However, radio quasars and GRBs, despite the aspirations of the authors, simply lack sharp features that allow their signals to be easily used to probe the intervening electrons in the IGM.

The situation can completely change with the recently discovered short radio bursts. The first such burst was reported by Lorimer et al. (2007), which is an intense (30 Jy) and shortduration $(5 \mathrm{~ms}$ ) burst at $1.4 \mathrm{GHz}$ (named as the Sparker in Kulkarni et al. 2014). Following the above discovery, Thornton et al. (2013) reported the finding of four short-duration bursts with an estimated all-sky rate of $10^{4}$ events day ${ }^{-1}$ (denoted as "fast radio bursts" or FRBs). Spitler et al. (2014) discovered one FRB in the Arecibo Pulsar ALFA Survey. These short radio bursts show DMs of order of a few hundred to a thousand, suggesting a substantial contribution from electrons in the IGM. Kulkarni et al. (2014) performed a thorough investigation of the Sparker and FRBs to explore possible constraints on sites or processes to explain such high DMs and concluded that they are of extragalactic origin, provided that the inferred DM arises as a result of propagation through cold plasma. A variety of models have been proposed for the progenitors of such short bursts (e.g., Popov \& Postnov 2010; Vachaspati 2008; Falcke \& Rezzolla 2014; Totani 2013; Zhang 2014; Lasky et al. 2014; Kashiyama et al. 2013; Kulkarni et al. 2014).
The pulse nature, the high rate, and the extragalactic origin make the Sparker-like events and FRBs well suited for being used as a potentially powerful probe to the IGM. Since the discovery of the short-duration bursts, DM measurements have been proposed to probe missing baryons around halos of galaxies (McQuinn 2014), study the baryon content in the IGM (Deng \& Zhang 2014), and constrain cosmology and the equation of state of dark energy (Gao et al. 2014; Zhou et al. 2014).

In this paper, we explore further potential applications of a Sparker-like population or FRBs in probing the IGM (for simplicity, hereafter we refer to the Sparker-like events and FRBs collectively as FRBs). Specifically, we first point out the use of them to probe the era of He II reionizationand intergalactic magnetic field (Section 2) and then comment about the potential use of FRBs for detecting a cosmological population of massive compact halo objects, i.e., MACHOs (Section 3). Finally, we give a summary in Section 4.

\section{PROBING He II REIONIZATION AND IGM MAGNETIC FIELD}

In this section, we specifically focus on the potential use of FRBs to probe the era of He II reionization and IGM magnetic field.

He II reionization can be regarded as the last phase transition in the universe, after the major one related to hydrogen and He I reionization above $z \sim 6$. Stars are not hot enough to ionize $\mathrm{He}$ II with an ionization potential of $54.4 \mathrm{eV}$. However, there is some evidence that He II reionization occurred at $z \sim 3$ from the transmission of the He II Ly $\alpha$ forest and the temperature change of the IGM (see Furlaneto \& Oh 2008a, 2008b and references therein). The ionization can be caused by soft X-ray emission from activate galactic nuclei (AGNs) and hard ionizing photons from quasars. Thus, observations of He II reionization provide a new diagnostic of the buildup of the AGN and quasar population. The same observations also provide important clues to the structure and thermal evolution of the IGM, which is related to the missing baryons problem (e.g., Gnat 2011). Clearly, there 
is great value in using FRBs in our study of cosmology and AGNs/quasars.

For the purpose of computing the DM from the IGM, there are three effects on the propagation time, $t_{p}$, of a photon traveling through the IGM to reach the observer from a cosmological distance: the continuous change of the photon's frequency, $\omega$, due to the redshift of light; the change of the plasma frequency, $\omega_{p}^{2}=4 \pi n_{e}(z) e^{2} / m_{e}$, due to the change in the IGM electron density, $n_{e}(z)$, with redshift; and the time dilation effect. The first two effects lead to a change in the group velocity, $v_{g}=c\left(1-\omega_{p}^{2} / \omega^{2}\right)^{1 / 2}$, with redshift. The propagation time of a photon emitted at redshift $z$ seen by an observer at redshift 0 is then

$$
\begin{aligned}
t_{p} & =\int_{0}^{z} d z \frac{d l}{d z} \frac{1}{v_{g}}(1+z), \\
& =\int_{0}^{z} \frac{c d z}{(1+z) H(z)} \frac{1}{c}\left(1+\frac{1}{2} \frac{\omega_{p}^{2}}{\omega^{2}}\right)(1+z),
\end{aligned}
$$

where $H(z)=H_{0}\left[\Omega_{m}(1+z)^{3}+\Omega_{\Lambda}\right]^{1 / 2}$ is the Hubble constant at $z$, with $\Omega_{m}$ the matter density parameter and $\Omega_{\Lambda}=1-\Omega_{m}$ (assuming a spatially flat universe), and the last $(1+z)$ factor accounts for time dilation. The frequency, $\omega$, is related to the observed frequency, $\omega_{\text {obs }}$, through $\omega=(1+z) \omega_{\text {obs }}$.

The IGM electron density $n_{e}(z)$ can be expressed as

$$
\begin{aligned}
n_{e}(z) & =n_{0}(1+z)^{3}\left[(1-Y) f_{\mathrm{H} \text { II }}+\frac{1}{4} Y\left(f_{\mathrm{He} \text { II }}+2 f_{\mathrm{He} \text { III }}\right)\right], \\
& =n_{0}(1+z)^{3} f_{e}(z),
\end{aligned}
$$

where

$$
n_{0}=\frac{\Omega_{b} \rho_{c}}{m_{H}}=2.475 \times 10^{-7}\left(\frac{\Omega_{b} h^{2}}{0.022}\right) \mathrm{cm}^{-3}
$$

is the mean number density of nucleons at $z=0$. Here $\Omega_{b}$ is the baryon density in units of the $z=0$ critical density $\rho_{c}$ and $h$ is the $z=0$ Hubble constant in units of $100 \mathrm{~km} \mathrm{~s}^{-1} \mathrm{Mpc}^{-1}$. Since we assume to observe a large number of FRBs at each redshift, it is appropriate to use the mean density of the IGM in the calculation without worrying about the density fluctuations. In the expression, $Y \simeq 0.25$ is the mass fraction of helium, $f_{\mathrm{H} \text { II }}$ is the ionization fraction of hydrogen, and $f_{\mathrm{He}}$ II and $f_{\mathrm{He}}$ III are the ionization fractions of singly and doubly ionized helium. After helium reionization $(z \sim 2-3)$, we essentially have $f_{\mathrm{H} \text { II }}=1$, $f_{\mathrm{He} \text { II }}=0$, and $f_{\mathrm{He} \text { III }}=1$, which gives $f_{e} \simeq 0.88$ at low redshifts.

The observed DM is defined as

$$
\frac{d t_{p}}{d \omega_{\mathrm{obs}}}=-\frac{4 \pi e^{2}}{c m_{e} \omega_{\mathrm{obs}}^{3}} \mathrm{DM}
$$

In combination with Equation (1), we have

$$
\begin{aligned}
\mathrm{DM}= & n_{0} \frac{c}{H_{0}} \int_{0}^{z} \frac{d z(1+z) f_{e}(z)}{\sqrt{\Omega_{m}(1+z)^{3}+\Omega_{\Lambda}}}, \\
= & 1060 \mathrm{~cm}^{-3} \mathrm{pc}\left(\frac{\Omega_{b} h^{2}}{0.022}\right)\left(\frac{h}{0.7}\right)^{-1} \\
& \times \int_{0}^{z} \frac{d z(1+z) f_{e}(z)}{\sqrt{\Omega_{m}(1+z)^{3}+\Omega_{\Lambda}}} .
\end{aligned}
$$

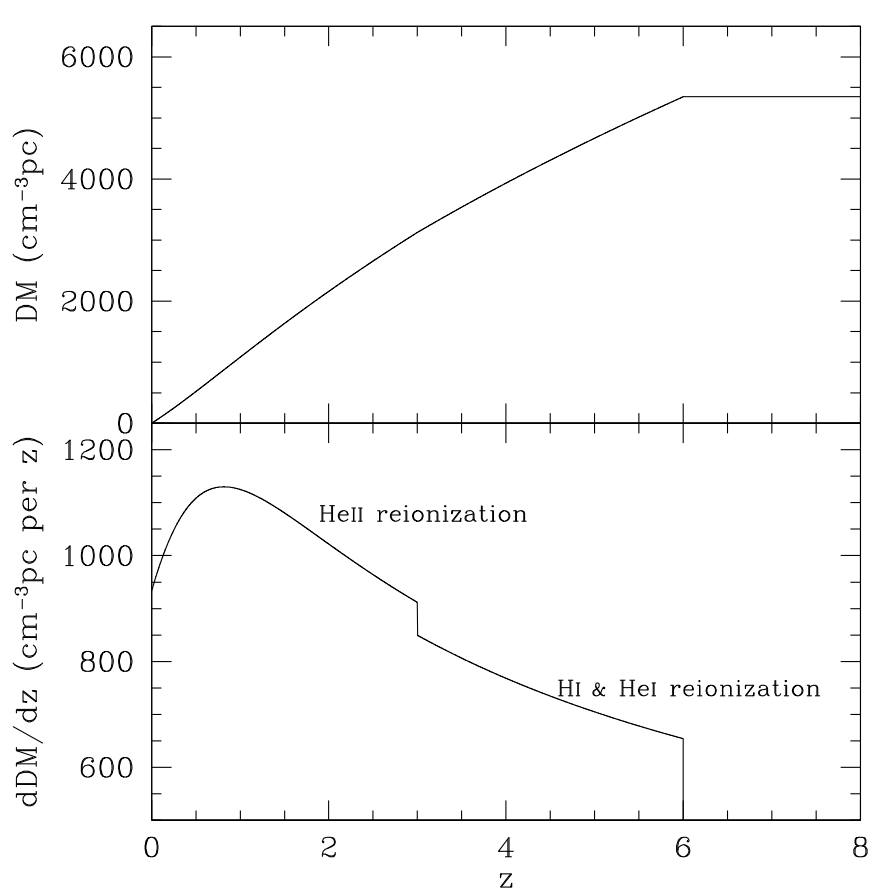

Figure 1. Illustration of using dispersion measure to probe the epoch of reionization of He II. Top and bottom panels show DM and its derivative as a function of redshift, respectively. A sharp $\mathrm{HI}$ and He I reionization at $z \sim 6$ and a sharp He II reionization at $z \sim 3$ are assumed.

For a constant $f_{e}$, the above integral can be approximated as

$$
\begin{aligned}
\mathrm{DM} \cong & 933 \mathrm{~cm}^{-3} \mathrm{pc}\left(\frac{f_{e}}{0.88}\right)\left(\frac{\Omega_{b} h^{2}}{0.022}\right)\left(\frac{h}{0.7}\right)^{-1} \\
& \times\left[\left(\frac{\Omega_{m}}{0.25}\right)^{0.1} a_{1}(x-1)+\left(\frac{\Omega_{m}}{0.25}\right) a_{2}\left(x^{2.5}-1\right)\right. \\
& \left.+\left(\frac{\Omega_{m}}{0.25}\right)^{1.5} a_{3}\left(x^{4}-1\right)\right]
\end{aligned}
$$

with $x=1+z, a_{1}=0.5372, a_{2}=-0.0189$, and $a_{3}=0.00052$. The accuracy of this approximation is better than $\sim 2 \%$ for $z<5$. At low redshifts, one can use the following approximation:

$$
\begin{aligned}
\mathrm{DM} \cong & 933 \mathrm{~cm}^{-3} \operatorname{pc}\left[z+\left(0.5-0.75 \Omega_{m}\right) z^{2}\right] \\
& \times\left(\frac{f_{e}}{0.88}\right)\left(\frac{\Omega_{b} h^{2}}{0.022}\right)\left(\frac{h}{0.7}\right)^{-1},
\end{aligned}
$$

which has a $5 \%$ accuracy up to $z=0.6$. For a constant $f_{e}$, the integral in Equation (5) shares some similarity with the expression of the luminosity distance $D_{L}$, with the $(1+z)$ factor pulled out of the integral in the latter. In terms of $D_{L}$, the integral can be approximated as

$$
\mathrm{DM} \cong n_{0} f_{e} D_{L}\left[1+0.932 z+\left(0.16 \Omega_{m}-0.078\right) z^{2}\right]^{-0.5},
$$

which has an accuracy $\lesssim 0.5 \%$ for $0<z<3$ with $0.25<$ $\Omega_{m}<0.35$.

As an illustration, the DM as a function of $z$ is displayed in Figure 1. This is an idealized plot since we assume a sharp He II reionization at $z \sim 3$. The reionization is better seen in the slope or derivative of the DM curve. The jump is about $8 \%$. Whether this jump will be seen or not will depend very strongly on the contribution to the DM of FRBs by the electrons in the host 
galaxies and whether FRBs can be found to redshifts as high as $z \sim 3$.

Similarly, we can obtain the rotation measure (RM). The Faraday rotation is

$$
\Delta \theta=\frac{2 \pi e^{3}}{m_{e}^{2} c^{2} \omega_{\mathrm{obs}}^{2}} n_{0} B_{0} \frac{c}{H_{0}} \int_{0}^{z} \frac{f_{e}(z) b_{\|}(z) d z}{\sqrt{\Omega_{m}(1+z)^{3}+\Omega_{\Lambda}}},
$$

where $b_{\|}(z) \equiv B_{\|}(z) / B_{0}$ is the line-of-sight magnetic field, $B_{\|}(z)$, in units of the local IGM magnetic field, $B_{0}$. We then have

$$
\begin{aligned}
\mathrm{RM}= & 8.61 \mathrm{rad} \mathrm{m}^{-2}\left(\frac{\Omega_{b} h^{2}}{0.022}\right)\left(\frac{h}{0.7}\right)^{-1}\left(\frac{B_{0}}{10 \mathrm{nG}}\right) \\
& \times \int_{0}^{z} \frac{f_{e}(z) b_{\|}(z) d z}{\sqrt{\Omega_{m}(1+z)^{3}+\Omega_{\Lambda}}} .
\end{aligned}
$$

At low redshifts, where we approximate $f_{e}=0.88$ and $b_{\|}=1$, the RM can be written as

$$
\begin{aligned}
\mathrm{RM} \cong & 7.57\left(z-0.75 \Omega_{m} z^{2}\right) \mathrm{rad} \mathrm{m}^{-2} \\
& \times\left(\frac{f_{e}}{0.88}\right)\left(\frac{\Omega_{b} h^{2}}{0.022}\right)\left(\frac{h}{0.7}\right)^{-1}\left(\frac{B_{0}}{10 \mathrm{nG}}\right) .
\end{aligned}
$$

So far, there are no accurate measurements for the magnetic field in the IGM with densities of the order of the mean density (see Kronberg et al. 2008). We note that the local IGM magnetic field would have a strength of $4\left(T_{\mathrm{IGM}} / 10^{4} \mathrm{~K}\right) \mathrm{nG}$ if energy equipartition is assumed. Radio-synchrotron radiation has been detected in the Coma supercluster (Kim et al. 1989), implying a field strength of $0.3-0.6 \mu \mathrm{G}$. RM measurements of FRBs can constraint the magnitude of IGM magnetic field and its evolution, providing clues on its origin.

In addition, if the DM and RM have a large contribution from a scattering screen, measurement of RM will provide a strong clue to the location of the scattering (and thus dispersion) screen. If the scattering arises in the IGM, then the RM from the IGM is less than $30 \mathrm{rad} \mathrm{m}^{-2}$ for $z \lesssim 0.3$ (Kronberg et al. 2008). A much larger RM can be produced if the screen is located in the host galaxy.

\section{PROBING INTERGALACTIC MACHOS}

Another potential use of FRBs is to constrain the existence of floating MACHO-like objects in the IGM via gravitational lensing (e.g., Gould 1992; Stanek et al. 1993; Marani et al. 1999).

A fortunate alignment of an intervening point-mass object of mass $M$ with an FRB will result in two images. The time delay for each image, with respect to a ray that arrives by the shortest path, is the sum of a geometric term and a gravitational delay term and is given by Narayan \& Bartelmann (1996) as

$$
t(\theta)=\frac{1+z_{l}}{c} \frac{4 G M}{c^{2}}\left(\frac{1}{2} \frac{\theta^{2}}{\theta_{\mathrm{E}}^{2}}-\ln |\theta|\right),
$$

where $z_{l}$ is the redshift of the lens, $\theta$ is the angular distance between the lens and the image, and $\theta_{\mathrm{E}}$ is the annular radius of the Einstein ring,

$$
\theta_{\mathrm{E}}=\sqrt{\frac{4 G M}{c^{2}} \frac{D_{l s}}{D_{l} D_{s}}}
$$

Here $D_{l}, D_{s}$, and $D_{l s}$ are the observer-lens, observer-source, and lens-source angular diameter distances, respectively. The positions of the two images are

$$
\theta_{ \pm}=\frac{1}{2}\left(b \pm \sqrt{b^{2}+4 \theta_{\mathrm{E}}^{2}}\right)
$$

where $b$ is the impact parameter (i.e., source-lens angular distance).

From Equations (12) and (14), the lensing time delay between the two images is

$$
\begin{aligned}
\Delta t_{l} & \equiv t\left(\theta_{-}\right)-t\left(\theta_{+}\right) \\
& =\frac{1+z_{l}}{c} \frac{4 G M}{c^{2}}\left[\frac{1}{2} u \sqrt{u^{2}+4}+\ln \left(\frac{\sqrt{u^{2}+4}+u}{\sqrt{u^{2}+4}-u}\right)\right],
\end{aligned}
$$

where $u \equiv b / \theta_{\mathrm{E}}$ is the impact parameter in units of the Einstein ring radius. For a typical value of $u=1$,

$$
\Delta t_{l}=41\left(1+z_{l}\right)\left(M / 1 M_{\odot}\right) \mu \mathrm{s} .
$$

The short timescales of FRBs make the effect of relative motions between the source, lens, and observer completely negligible (e.g., the fractional change in $u$ is of the order of $10^{-12}$ during a lensed millisecond FRB event at cosmological distances with relative motion of $100 \mathrm{~km} \mathrm{~s}^{-1}$ ). So unlike the case for Galactic MACHOs, we do not expect to observe microlensing light curves for MACHO-like objects in the IGM. However, the two images have different amplifications, $A_{ \pm}=1 / 2 \pm\left(u^{2}+2\right) /\left(2 u \sqrt{u^{2}+4}\right)$, and the time delay ensures they also have different phases. Therefore, the two images undergo constructive and destructive interference. The total amplification is

$$
A(\omega)=\frac{u^{2}+2}{u \sqrt{u^{2}+4}}+\frac{2}{u \sqrt{u^{2}+4}} \cos \left(\omega \Delta t_{l}\right),
$$

where $v=\omega /(2 \pi)$ is the frequency in the observer's frame. The spectrum of the lensed FRB will thus consist of maxima and minima with the separation of two maxima (or minima) being $\Delta v=\Delta t_{l}^{-1}$, which is tens of $\mathrm{kHz}$ for $M \sim 1 M_{\odot}$ and $u \sim 1-$ well within the reach of current technology. Note that $\Delta v$ is independent of the observing frequency, if the time delay is purely caused by lensing. However, in general $\Delta t_{l}$ in Equation (17) should be replaced by the sum of all possible time delays. There could be dispersive delay due to the rays suffering different DM or delay due to multipath propagation from a scattering screen. For FRBs, the latter effect destroys the coherence ${ }^{6}$ of the rays. For this reason, observing at higher frequencies (i.e., $\gtrsim 5 \mathrm{GHz}$ ) is highly desirable. Furthermore, a matched-filter approach ${ }^{7}$ can additionally improve the detection.

Finally, we can estimate the optical depth for lensing (e.g., Narayan \& Bartelmann 1996). For a proper number density $n(z)$ of lenses with mass $M$ in a spatially flat universe, the optical depth for lensing to sources at redshift $z_{s}$ is

$$
\begin{aligned}
\tau\left(z_{s}\right) & =\int_{0}^{z_{s}} n(z) \pi\left(D_{l} \theta_{E}\right)^{2} d D_{c} /(1+z) \\
& =\frac{4 \pi G M}{c^{2} D_{s}} \int_{0}^{z_{s}} n(z) D_{l s} D_{l} d D_{c} /(1+z),
\end{aligned}
$$

\footnotetext{
6 Equivalently, the de-coherence is the size of the scattered disk exceeding the image separation with the attendant loss of visibility function.

7 Naturally obtained via the cross-correlation function in an XF-type interferometer.
} 
where the angular diameter distances are $D_{s}=D_{A}\left(0, z_{s}\right), D_{l}=$ $D_{A}(0, z), D_{l s}=D_{A}\left(z, z_{s}\right)$, and $D_{A}\left(z_{1}, z_{2}\right)=\int_{z_{1}}^{z_{2}} d D_{c} /\left(1+z_{2}\right)$, with $d D_{c}=c d z / H(z)$ the comoving distance element. In the case of proper number density evolving as $n_{0}(1+z)^{2}$ (i.e., comoving number density evolving as $\left.n_{0} /(1+z)\right)$, the optical depth can be put in a form similar to the result in the static Euclidean space,

$$
\tau=\frac{2 \pi}{3} \frac{G \rho_{l, 0}}{c^{2}} D_{c}^{2} \simeq 0.014 \Omega_{l}\left(D_{c} / 1 \mathrm{Gpc}\right)^{2},
$$

where $\rho_{l, 0}=n_{0} M$ is the mass density of lenses at $z=0$ and $\Omega_{l}$ is this mass density in units of the $z=0$ critical density of the universe. In the case of a constant comoving number density, the result is about 44\% higher than that in Equation (19) for $z_{s}=1$.

\section{SUMMARY AND DISCUSSION}

The pulse nature, the high rate, and the extragalactic origin make FRBs ideal for probing the IGM. In this paper, we present potential applications of FRBs to probe He II reionization, IGM magnetic field, and MACHO-like objects in the IGM.

For these applications, a large population of FRBs is necessary. The He II reionization causes a small change in the DM. It leads to a $\sim 8 \%$ jump in the differential DM across the He II reionization epoch. At least a few hundred FRBs around $z \sim 2-3$ are needed to detect such a change. Our Galaxy, FRB host galaxies, and any intervening galaxies or clouds with free electrons can add scatter in the DM. Clearly, more FRBs and searching for host galaxies are desired to constrain and understand such a scatter. It is likely that we need thousands of FRBs around $z \sim 2-3$ to learn about the He II reionization from the DM measurements.

The probe of IGM magnetic field with FRBs could be more challenging, given that its strength is likely of the order of $\mathrm{nG}$ while that inside a galaxy is of the order of $\mu \mathrm{G}$. The RM from a typical galaxy is about $812 \mathrm{rad} \mathrm{m}^{-2}\left(n_{e} / \mathrm{cm}^{-3}\right)\left(B_{\|} / \mu G\right)(l / \mathrm{kpc})$ (with $l$ the path length). The RM from IGM to $z=1$ is only $6 \mathrm{rad} \mathrm{m}^{-2}$ for an IGM magnetic field of $10 \mathrm{nG}$ according to Equation (11). If the scatter in the galaxy-caused RM is of the same order of $\sim 800 \mathrm{rad} \mathrm{m}^{-2}$, tens of thousands of FRBs are needed to clearly map out the redshift evolution of RM caused by the IGM magnetic field.

For the MACHO-like objects in the IGM, the upper bound for their density parameter $\Omega_{l}$ is $\Omega_{m}$. If they are of baryonic origin (e.g., stellar remnants), which may be more likely, the upper bound is then $\Omega_{b}$. According to Equation (19), we expect the lensing optical depth to be (much) less than $6 \times 10^{-4}$ to a distance of $1 \mathrm{Gpc}$, which requires at least tens of thousands of FRBs to discover the events with the lensing signal we point out.

The current estimated all-sky rate of FRBs is $10^{4}$ events day $^{-1}$ (Thornton et al. 2013). Therefore, with well-designed and dedicated FRB surveys, all the above requirements of a large statistical sample of FRBs are not demanding at all. Such surveys would provide an invaluable opportunity to advance our understanding of the IGM.

We thank Shude Mao for useful comments. Z.Z. was partially supported by NSF grant AST-1208891 and NASA grant NNX14AC89G. M.J. acknowledges the support of the Washington Research Foundation through its Data Science Chair and the University of Washington Provost's Initiative in Data-Intensive Discovery. E.O.O. is incumbent of the Arye Dissentshik career development chair and is grateful to support by grants from the Willner Family Leadership Institute Ilan Gluzman (Secaucus, NJ), Israeli Ministry of Science, Israel Science Foundation, Minerva and the I-CORE Program of the Planning and Budgeting Committee, and The Israel Science Foundation. S.R.K. thanks the hospitality of the Institute for Advanced Study (IAS). The sylvan surroundings and verdant intellectual ambiance of IAS resulted in a fecund mini-sabbatical stay (Fall 2007).

\section{REFERENCES}

Deng, W., \& Zhang, B. 2014, ApJL, 783, L35

Falcke, H., \& Rezzolla, L. 2014, A\&A, 562, A137

Furlanetto, S. R., \& Oh, S. P. 2008a, ApJ, 682, 14

Furlanetto, S. R., \& Oh, S. P. 2008b, ApJ, 681, 1

Gao, H., Li, Z., \& Zhang, B. 2014, ApJ, 788, 189

Ginzburg, V. L. 1973, Natur, 246, 415

Gnat, O. 2011, ApJ, 729, 82

Gould, A. 1992, ApJL, 386, L5

Haddock, F. T., \& Sciama, D. W. 1965, PhRvL, 14, 1007

Inoue, S. 2004, MNRAS, 348, 999

Ioka, K. 2003, ApJL, 598, L79

Kashiyama, K., Ioka, K., \& Mészáros, P. 2013, ApJL, 776, L39

Kim, K.-T., Kronberg, P. P., Giovannini, G., \& Venturi, T. 1989, Natur, 341, 720

Kronberg, P. P., Bernet, M. L., Miniati, F., et al. 2008, ApJ, 676, 70

Kulkarni, S. R., Ofek, E. O., Neill, J. D., Zheng, Z., \& Juric, M. 2014, ApJ, 797, 70

Lasky, P. D., Haskell, B., Ravi, V., Howell, E. J., \& Coward, D. M. 2014, PhRvD, 89, 047302

Lipunova, G. V., Panchenko, I. E., \& Lipunov, V. M. 1997, NewA, 2, 555

Lorimer, D. R., Bailes, M., McLaughlin, M. A., Narkevic, D. J., \& Crawford, F. 2007, Sci, 318, 777

Marani, G. F., Nemiroff, R. J., Norris, J. P., Hurley, K., \& Bonnell, J. T. 1999, ApJL, 512, L13

McQuinn, M. 2014, ApJL, 780, L33

Narayan, R., \& Bartelmann, M. 1996, arXiv:astro-ph/9606001

Palmer, D. M. 1993, ApJL, 417, L25

Popov, S. B., \& Postnov, K. A. 2010, in Evolution of Cosmic Objects through their Physical Activity, ed. H. A. Harutyunian, A. M. Mickaelian, \& Y. Terzian (Yerevan: Publishing House of NAS RA), 129

Spitler, L. G., Cordes, J. M., Hessels, J. W. T., et al. 2014, ApJ, 790, 101

Stanek, K. Z., Paczynski, B., \& Goodman, J. 1993, ApJL, 413, L7

Thornton, D., Stappers, B., Bailes, M., et al. 2013, Sci, 341, 53

Totani, T. 2013, PASJ, 65, L12

Vachaspati, T. 2008, PhRvL, 101, 141301

Weinberg, S. 1972, Gravitation and Cosmology: Principles and Applications of the General Theory of Relativity (New York: Wiley-VCH)

Zhang, B. 2014, ApJL, 780, L21

Zhou, B., Li, X., Wang, T., Fan, Y.-Z., \& Wei, D.-M. 2014, PhRvD, 89,107303 